\title{
Statistically Quantifying the Uncertainty in Strength of Marine Clay Stabilized by a Type of Portland-Fly Ash Cement
}

\author{
Zhi-Ren Zhu, ${ }^{1}$ Qiang-Qiang Cheng, ${ }^{2,3}$ Yang-Yang Chen, ${ }^{4}$ Elton J. Chen $\mathbb{D}^{4},{ }^{4}$ and Yuan Su ${ }^{4}$ \\ ${ }^{1}$ College of Civil Engineering, Hunan University, Changsha 410012, China \\ ${ }^{2}$ Jiangsu Vocational Institute of Architectural Technology, Xuzhou, China \\ ${ }^{3}$ State Key Laboratory of Coal Resources and Safe Mining, China University of Mining \& Technology, Xuzhou, China \\ ${ }^{4}$ School of Civil and Hydraulic Engineering, Huazhong University of Science and Technology, Wuhan, China \\ Correspondence should be addressed to Elton J. Chen; eltonjchen@hust.edu.cn
}

Received 3 October 2020; Revised 6 January 2021; Accepted 22 January 2021; Published 23 February 2021

Academic Editor: Yong Liu

Copyright ( $) 2021$ Zhi-Ren Zhu et al. This is an open access article distributed under the Creative Commons Attribution License, which permits unrestricted use, distribution, and reproduction in any medium, provided the original work is properly cited.

\begin{abstract}
This study deals with the variation in strength of clays stabilized by a special binder-the CEM II/B-V type of Portland-fly ash cement. Two sources of uncertainties were examined, that is, the uncertainty in cement fraction in the binder, and the nonuniformity in binder concentration resulted from the process of mixing binder slurry with in situ clays. These two uncertainties were quantitatively described as two random variables, and the analyses were conducted through the Monte Carlo simulation method. Results indicate that the strength is insensitive to the cement fraction in the binder but is sensitive to the binder concentration; the variation of the latter even results in an amplified variation of strength. The variation of binder concentration is proposed to be linked up with the variation of strength through the operational parameter of blade rotation number. By doing so, the results could benefit designers in quality control prior to the construction of soil stabilization.
\end{abstract}

\section{Introduction}

Soil stabilization is necessary prior to the constructions of many geotechnical projects in marine clay. The stabilization can be realized by adding cementitious binders or other admixtures (e.g., lime, fly ash, and bitumen) into natural clays with the deep soil mixing or jet-grouting techniques. Ordinary Portland cement (OPC) is a common binder, with which the stabilized soil can gain strength in a relatively fast manner; around $80 \%$ strength can be reached within a 28 day curing period. This feature enables the OPC being used in projects with a short construction period. However, many projects have a long construction period; for instance, when applying a cement-admixed slab in a deep excavation to resist the lateral displacement ([1]), the excavation may last half a year after the completion of the slab. In this circumstance, a low strength gaining rate is allowable. Xiao et al. [2] investigated the effect of fly ash on the strength gaining rate by using a new binder, that is, the CEM II/B-V type of Portland-fly ash cement (PFC), which is a mixture of
OPC with a certain amount of siliceous fly ash ([3]). Figure 1 illustrates the PFC in macrolevel and microlevel. Figure 2 reinterprets the data from Xiao et al.'s [2] work, which shows that the strength of OPC-stabilized soil with fly ash exceeds that without fly ash when the curing period is 90 days or more. Thus, if a project lasts longer than 3 months before the stabilized soil to be mobilized, the PFC is likely to be a better binder over pure OPC in terms of strength gain.

A few studies on the PFC-stabilized clays can be found $([2,4,5])$, which were mainly based on laboratory tests with cautiously prepared samples and standard procedures in testing. As a result, the strength variation of laboratory samples is much less than that observed in the field. For instance, Xiao et al. [2] found that the coefficient of variation (COV) of the unconfined compressive strength (UCS) of PFC-stabilized clay samples prepared in laboratory is generally less than 0.05 . This level of COV is negligible when compared to that reported from deep cement mixing projects; the latter is often as high as 0.4 to 0.6 ([6]). This discrepancy implies a gap between laboratory studies and 


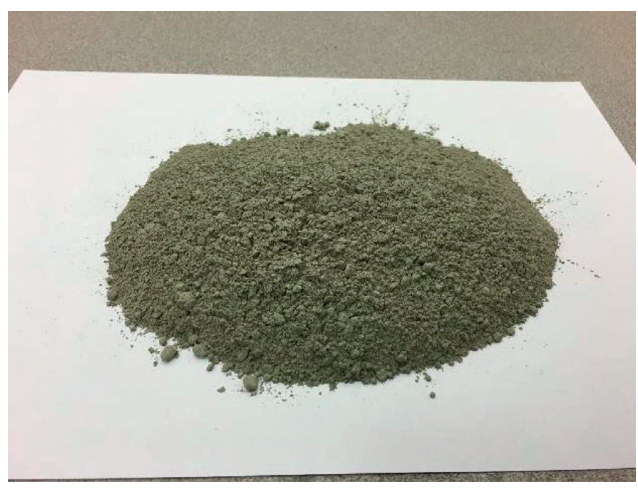

(a)

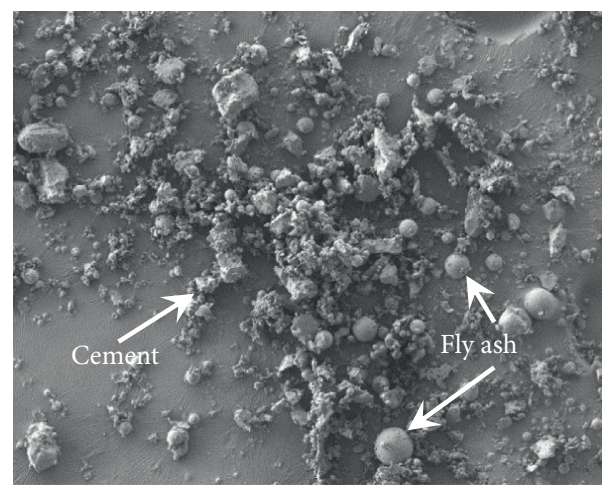

(b)

Figure 1: (a) Macrolevel and (b) microlevel photos of Portland-fly ash cement (prototype size of the image in (b) is around $2 \mathrm{~mm} \times 2 \mathrm{~mm}$ ).

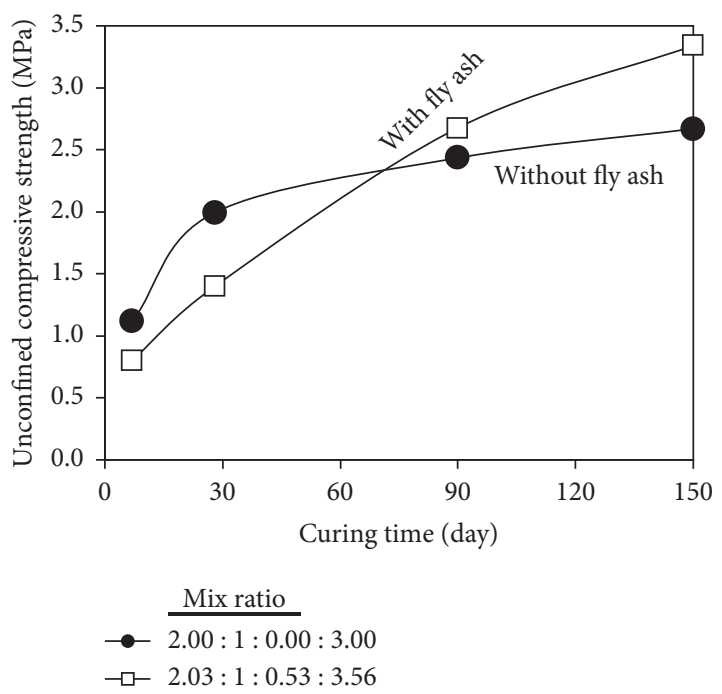

FIgURE 2: Unconfined compressive test results of two sets of samples with $100 \%$ water content but different mix ratios (mix ratio is defined as the mass ratio of soil, ordinary Portland cement, fly ash, and water, that is, $\mathrm{S}: \mathrm{C}: \mathrm{F}: \mathrm{W})$.

engineering practices. It may be attributable to the uncertainties encountered in construction. Significant nonuniformity can be introduced during the process of mixing the binder with in situ clay. This type of nonuniformity has been widely reported (e.g., [7-9]). On the other hand, PFC is a mixture of cement and fly ash, and the cement fraction of PFC is specified as a range of $65 \%-79 \%$ in BS EN $1971: 2000$ [3]. The variation in cement fraction in the binder is likely to further increase the variation in strength.

In the current study, these two sources of uncertainties are considered to link laboratory results with engineering applications. The relationship in the coefficient of variation (COV) between laboratory and field data is established. With this relationship, a rule-of-thumb estimation of the variation in strength can be obtained for construction quality control.

\section{Strength Prediction Model}

Based on a large number of experimental tests, Xiao et al. [2] concluded that the UCS of PFC-stabilized clay (denoted as $q_{u}$ ) is contributed by the OPC component (denoted as $q_{u c}$ ) and the fly ash component (denoted as $q_{u f}$ ):

$$
q_{u}=q_{u c}+q_{u f}
$$

in which $q_{u c}$ and $q_{u f}$ are in the units of $\mathrm{kPa}$.

$$
\begin{aligned}
& q_{u c}=44000 \times \frac{1+0.36 \times\left(\left(M_{S}+M_{F}\right) / M_{C}\right)+\left(0.36 \times\left(\left(M_{S}+M_{F}\right) / M_{C}\right)\right)^{2}}{\left(\left(M_{W 1}+M_{W 2}\right) / M_{C}\right)^{3.1}} \times\left(1-\frac{1}{1+(1174 t / 44000)^{0.52}}\right), \\
& q_{u f}=85000 \times \frac{1+0.36 \times\left(\left(M_{S}+M_{C}\right) / M_{F}\right)+\left(0.36 \times\left(\left(M_{S}+M_{C}\right) / M_{F}\right)\right)^{2}}{\left(\left(M_{W 1}+M_{W 2}-0.4 M_{C}\right) / M_{C}\right)^{3.1}} \times\left(1-\frac{1}{1+(1150 t / 85000)^{2.5}}\right),
\end{aligned}
$$

where $t$ is the curing period in the units of day, and the phase components and mix ratio are defined in Figure 3.
Equations (2) and (3) are transformed from a relationship between the unconfined compressive strength 


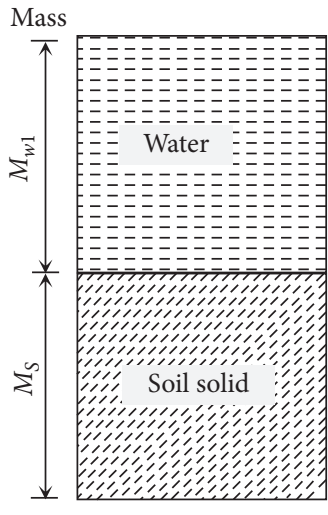

Natural soil

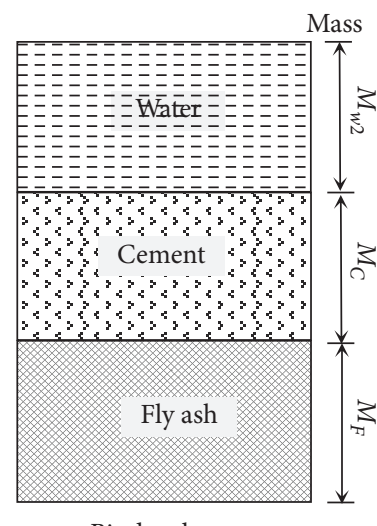

Binder slurry

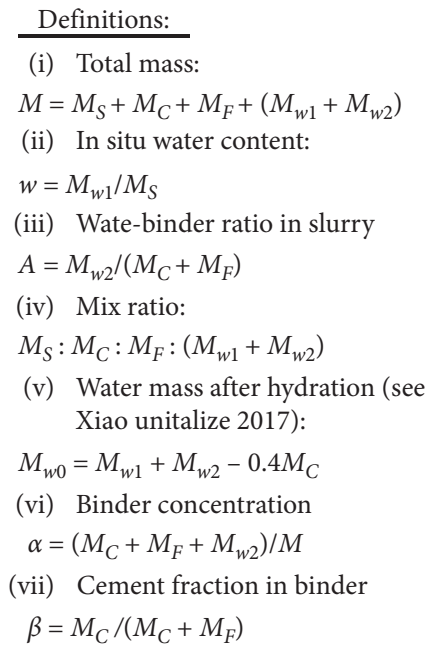

Figure 3: Illustration of components from a PFC-stabilized soil and definition of symbols used in the current study (PFC, Portland-fly ash cement; $M_{s}$ is the mass of solid soil in natural soil; $M_{c}$ is the mass of cement in blinder slurry; $M_{F}$ is the mass of fly ash in blinder slurry; $M_{w 1}$ is the mass of water in natural soil; and $M_{w 2}$ is the mass of water in blinder slurry).

$q_{u 0}$, mix ratio, and curing time $t$ is proposed by Xiao et al. $[2]$.

$$
q_{u 0}=q_{\infty}\left[1-\frac{1}{1+\left(\alpha t / q_{\infty}\right)^{r}}\right]\left\{\frac{1+m\left(1 / A_{w}\right)+\left[m\left(1 / A_{w}\right)\right]^{2}}{(W / C)^{n}}\right\},
$$

in which $A_{w}=$ cement content, $W=$ mass of water, and $C=$ mass of cement; $\alpha$ is related to the rate of short-term strength gain, whereas $r$ is related to the rate of long-term strength gain. By adjusting $m$ to obtain a best straight-line fit, parameters $m, q_{0}$, and $n$ can be determined. Table 1 summarizes the values of parameters in equation (4) for the specimen that consists of OPC component and fly ash component which is determined by Xiao et al. [2].

In the current study, a series of unconfined compression tests were conducted to verify equation (1). The mix ratio was set at $2: 0.655: 0.345: 3\left(M_{S}: M_{C}: M_{F}:\left(M_{W 1}+M_{W 2}\right)\right)$. The test results are shown in Figure 4, where Figures 4(b) and $4(\mathrm{c})$ show the failure pattern of compressed samples with 28-day and 90-day curing periods, respectively. For samples with 28 days of curing, the development and extension of fractures are mainly distributed in the upper zone which is about the $20-30 \%$ of the compressed sample. These continuous cracks compose a closed network and eventually contribute to the breakage of integrity of the sample. Conversely, on the part of the sample which cured for 90 days, the occurrence of failure is often accompanied by fragments peeling and the fractures apparently extend to the middle and bottom zones of the sample. Several main cracks across the whole sample in the end of the compression process is the significant reason for the failure of sample. Although no apparent shear bands were observed, which commonly occurs in unconfined compression tests, the test results are generally consistent with the prediction model of equation (1).
TABLE 1: Summary of parameters in equation (4).

\begin{tabular}{lcc}
\hline Component & Parameter & Value \\
\hline \multirow{3}{*}{ OPC } & $m_{1}$ & 0.36 \\
& $n_{1}$ & 3.1 \\
& $q_{\infty 1}$ & 44,000 \\
& $\alpha_{1}$ & 1,174 \\
& $r_{1}$ & 0.52 \\
\hline \multirow{3}{*}{ Fly ash } & $m_{2}$ & 0.36 \\
& $n_{2}$ & 3.2 \\
& $q_{\infty 2}$ & 85,000 \\
& $\alpha_{2}$ & 1,150 \\
& $r_{2}$ & 2.5 \\
\hline
\end{tabular}

\section{State Variables}

3.1. Soil Mixing. Figures 5(a) and 5(b) show soil-cement samples used in the laboratory and cored from a real project, respectively. As can be seen from Figure 5(b), much more nonuniformity can be observed in the cored samples, and some portions even remain untreated. This kind of nonuniformity is resulted from the soil mixing process. For this reason, a state variable $\alpha$ is defined (Figure 3 ) to quantify this nonuniformity. If $\alpha=0$, the admixture is pure natural soils; while if $\alpha=1$, the admixture is pure binder slurry.

3.2. Cement Fraction in PFC Binder. The binder of PFC (i.e., CEM II/B-V type of cement) is specified by BS EN 1971: 2000 [3], with a cement fraction being 65-79\%; equivalently, the fly ash mass fraction is $21-35 \%$. The cement fraction is also likely to vary during the process of blending cement with fly ash. As a result, since the cement and fly ash contribute to the strength in different manners (Figure 4), the resultant strength is likely to vary. To examine this effect, the cement mass fraction (denoted as $\beta$ ) is herein considered as another state variable. 


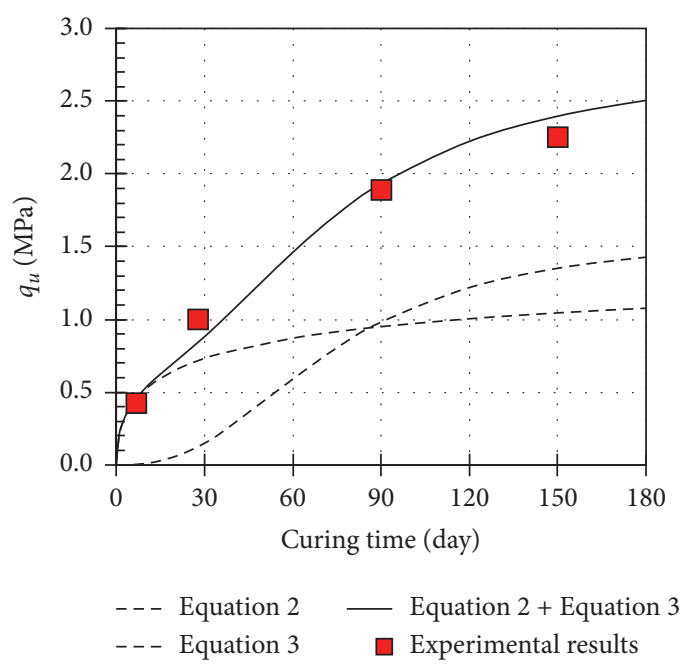

(a)

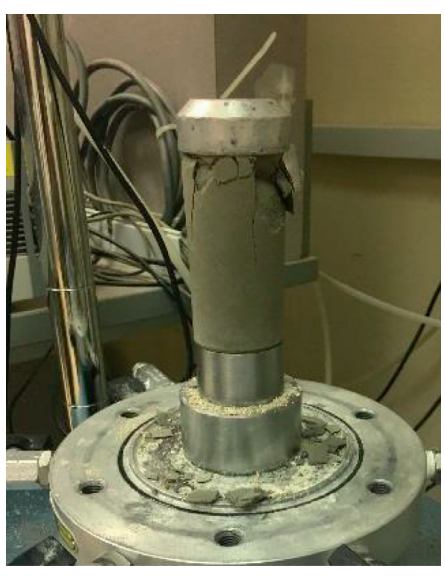

(b)

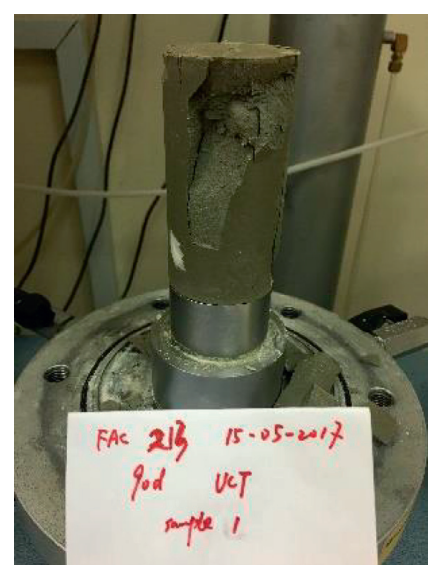

(c)

FIGURE 4: (a) Validation of the strength prediction model, and (b) and (c) samples after the unconfined compression test with 28-day and 90day curing periods, respectively.

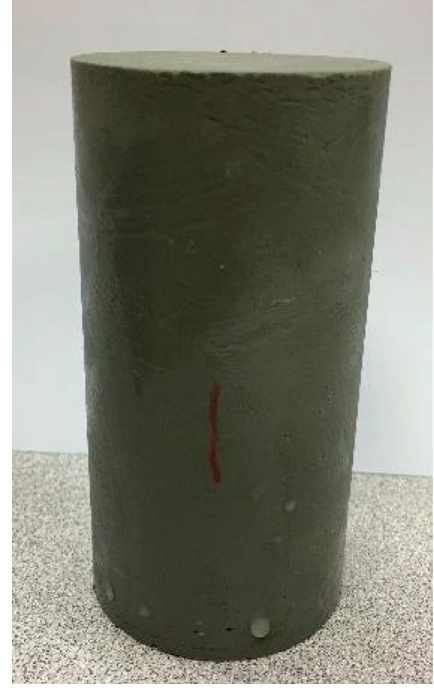

(a)

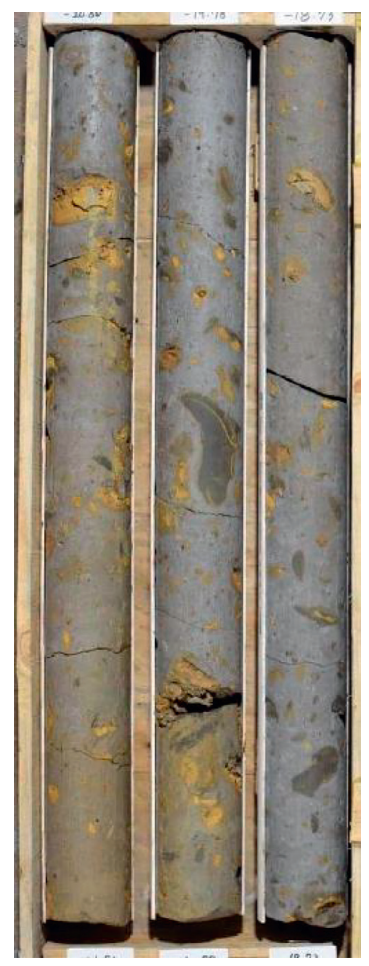

(b)

Figure 5: (a) A sample prepared in laboratory and (b) cored samples from a real project.

Figure 6 shows the resultant UCS under different levels of $\alpha$-value and $\beta$-value; their relationship is derived in Appendix A. The contribution of fly ash is insignificant when the curing period is less than 28 days, whereas its effect on strength becomes increasingly significant thereafter. Thus, in order to maximize its strength potential, a long curing period should be provided when using PFC as a stabilization binder.
3.3. Statistical Parameters. In this section, the state variables $\alpha$ and $\beta$ are treated as random variables to examine the statistical characteristics of the strength of PFC-stabilized clays.

The range of $\alpha$ is physically restricted at $[0,1]$. The mean value of $\alpha$ is prescribed by the designers-it depends on the dosage of cement. The mean value of $\alpha$ in samples tested in this study (Figure 4 ) is 0.33 . This mean value is adopted in 

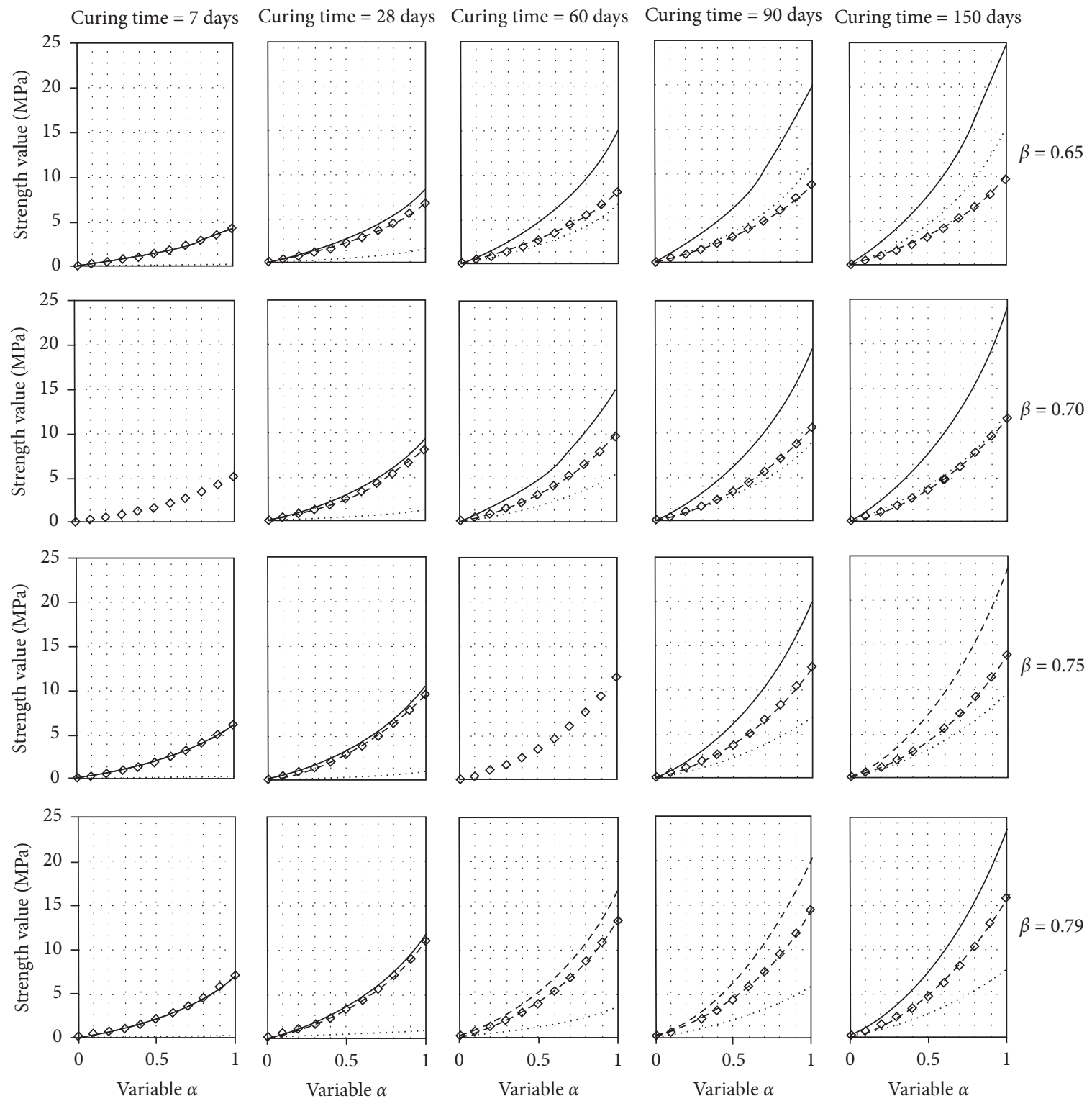

FIGURE 6: Resultant unconfined compressive strength (UCS) predicted under various combinations of binder concentration ( $\alpha$ ) and cement fraction in the binder $(\beta)$. Dashed curves with square marks: strength contributed from the OPC component (equation (2)); dashed curves: strength contributed from the fly ash component (equation (3)); solid curves: UCS of PFC-stabilized clay (equation (2) plus equation (3)).

the simulations hereafter, so that the simulation results can be compared to the laboratory test results. The COV of $\alpha$ can be inferred from the blade rotation number; the latter is an operational parameter of the mixing process when adding binder slurry into natural clays. Chen et al. [10] extensively examined the relationship between the COV of $\alpha$ and the blade rotation number. They observed that when the density of binder slurry approximates that of natural clays, the mixing uniformity can be enhanced, and they proposed the following formula based on centrifugal modelling test results:

$$
\delta=0.102+0.585 T^{-0.335},
$$

where $\delta$ denotes the $\operatorname{COV}$ of $\alpha$; Tdenotes the blade rotation number. For simplicity, $T=200$ is first adopted as a reference value, that is, $\delta=0.2$ based on equation (5). Up until this point, four parameters of the random variable $\alpha$ can be determined, namely, lower and upper bands, mean value, and COV. With these four parameters, a beta distribution can be determined for $\alpha$ ([11]).

On the other hand, since the main variability of $\beta$ results from its range specified by the BS code [3], the probability type of $\beta$ is simply assumed as the uniform distribution over the interval $[0.65,0.79]$. As will be shown later, the results are insensitive to the variation in $\beta$.

Figure 7 shows the probability density functions of $\alpha$ and $\beta$ for the reference case, and the specific statistical parameters of these two random variables are listed in Table 2.

\section{Results}

Figure 8 shows the effects of $\alpha$ and $\beta$ on the strength with the Monte Carlo simulation method [12]. The curing period is 


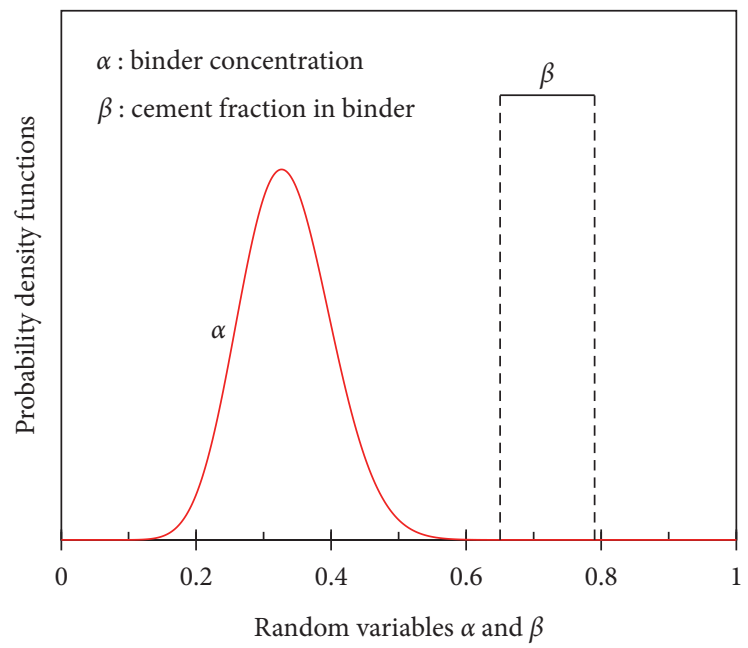

Figure 7: Probability density functions of $\alpha$ and $\beta$ ( $\alpha$, binder concentration; $\beta$, cement fraction in the binder).

TABLE 2: Statistical parameters of reference case.

\begin{tabular}{lccccc}
\hline Parameter & Symbols & Distribution type & Mean & COV & Range \\
\hline Binder concentration & $\alpha$ & Beta distribution & 0.33 & 0.2 & $0-1$ \\
Cement fraction in the binder & $\beta$ & Uniform distribution & - & - & $0.65-0.79$ \\
\hline
\end{tabular}

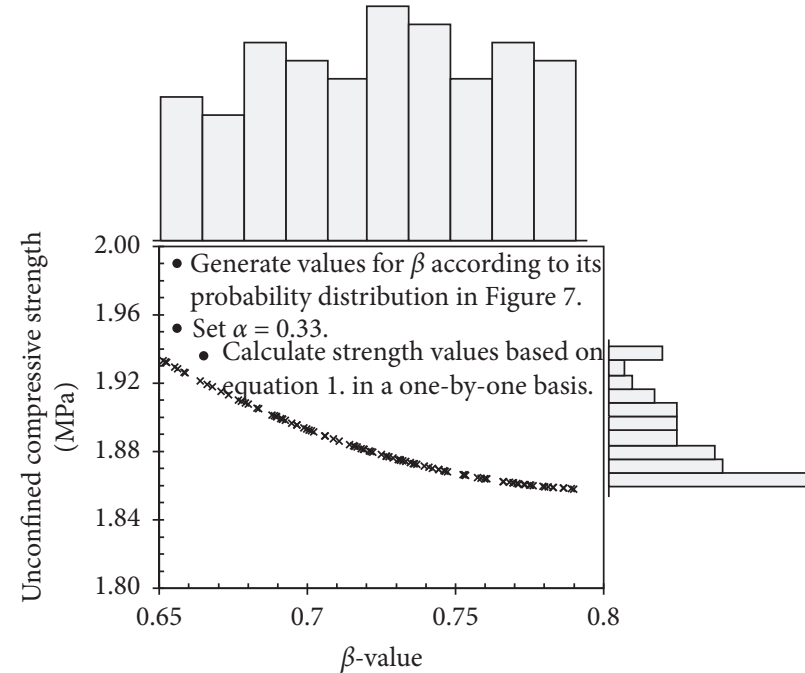

(a)

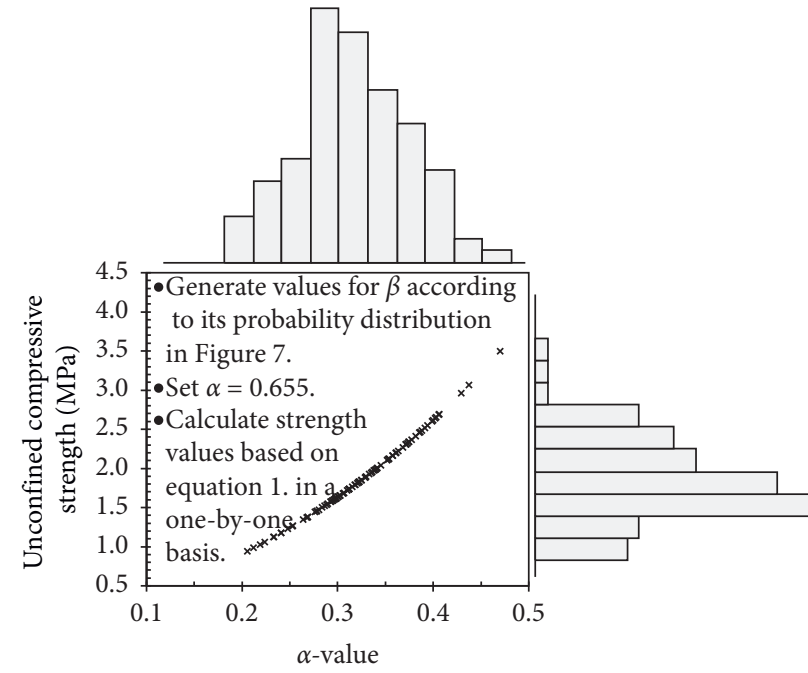

(b)

FIGURE 8: Illustrations of the Monte Carlo simulation method to examine the effect of variation in (a) $\beta$ and (b) $\alpha$ on unconfined compressive strength $(\alpha$, binder concentration; $\beta$, cement fraction in the binder).

adopted as 90 days; nevertheless, the results are extendable to other curing periods by considering the time-dependent factor ([2]). Figure 8(a) indicates that the variation in strength is relatively small; the maximum difference among the strength is less than $100 \mathrm{kPa}$. This observation implies that the strength function equation (1) is insensitive to $\beta$. This insensitivity can also be observed from Figure 6; the resultant effect of fly ash and cement on strength is insensitive to $\beta$ when the curing period is greater than 90 days, despite equations (2) and (3) separately vary with $\beta$. By contrast, Figure 8(b) shows that the strength varies significantly when $\alpha$ varies. Thus, the variation in the binder concentration $\alpha$ dominates the variation in strength. For these reasons, only the variation in $\alpha$ will be examined hereafter.

Parametric studies on $\alpha$ is conducted to evaluate the COV of field data. This is performed with the Monte Carlo simulations, and the results are shown in Figure 9. As can be seen from this figure, the two types of COVs can be bridged with the blade rotation number. The predicted strength COV 


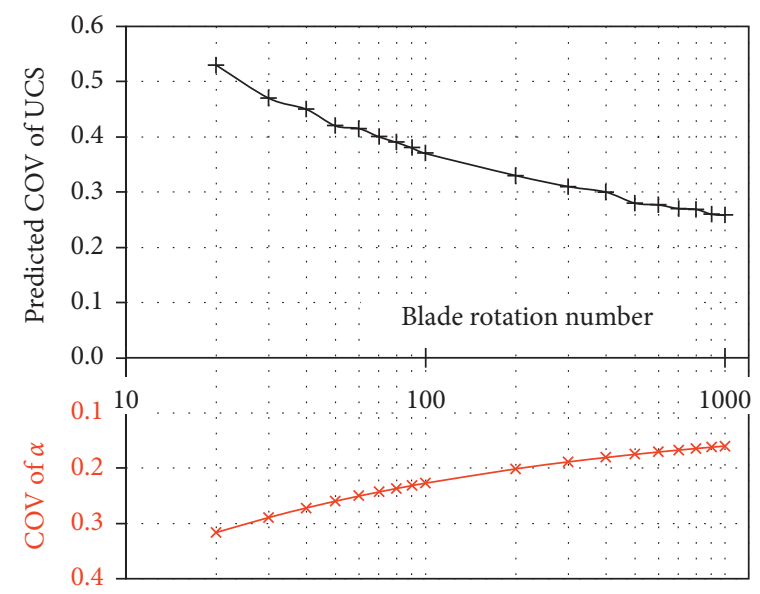

FIGURE 9: Prediction of coefficient of variation (COV) in unconfined compressive strength (UCS).

TABLE 3: Statistical and deterministic results of unconfined compressive strength.

\begin{tabular}{lccccc}
\hline \multicolumn{2}{c}{ Binder concentration $(\alpha)$} & \multicolumn{2}{c}{ Unconfined compressive strength $\left(q_{u}\right)$} & & Remarks \\
Mean & COV & Mean & Medium & COV & Deterministic case \\
\hline 0.33 & 0 & $1930 \mathrm{kPa}$ & - & - & Reference case \\
0.33 & 0.2 & $1985 \mathrm{kPa}$ & $1917 \mathrm{kPa}$ & 0.328 & \\
\hline
\end{tabular}

is greater than the COV of $\alpha$. As listed in Table 3, when the COV of $\alpha$ is 0.2 , the COV of strength is 0.328 . This implies that the nonuniformity during mixing has an amplifying effect on the strength variation. The amplifying effect is due to the nonlinearity of the strength prediction model of equation (1). By comparing the deterministic case and the random analysis case in Table 3, one can observe that the existence of variation in strength leads to a greater mean strength in result, while the medium value appears yield a more conservative estimation of the strength. This observation is consistent with Zheng et al.'s [13] work.

The predicted COV of UCS is a decreasing function of the blade rotation number, which is consistent with field observations reported by Fujii et al. [14] and Larsson et al. [15] in deep mixing projects. Fujii et al. [14] reported that, when the blade rotation number increases from 100 to 450 , the measured COV of UCS decreases from 0.8 to 0.16 . Larsson et al. [15] analysed the strength of deep mixing columns stabilized by lime-cement and found that the measured COV of strength decreases from around 0.6 to 0.1 when the blade rotation number increase from around 50 to 800 . As a result, Figure 9 is likely to provide a rule-of-thumb estimation of the $\mathrm{COV}$ in strength prior to the construction of a deep mixing project. Figure 9 and Table 3 could also serve as an extension work and lab-study support to Chen et al. [16] for the randomness of construction material properties and Pan et al. [17] for the uncertain factors in underground constructions.

\section{Conclusions}

A few laboratory studies can be found on the topic of applying Portland-fly ash cement as a binder for stabilization of marine clays, whereas these studies are mainly on the average level. In this study, the variation in strength of clays stabilized by the CEM II/B-V type of Portland-fly ash cement has been considered. The cement fraction of this type of cement is specified from $65 \%$ to $79 \%$. Two sources of uncertainties were examined, that is, the uncertainty in cement fraction of the binder, and the nonuniformity of binder concentration resulted from the mixing process of binder slurry with in situ clays. These two uncertainties were quantitatively assessed as two random variables, and the analyses were conducted through the Monte Carlo simulations. The results indicate that the strength of stabilized clays is insensitive to the cement fraction within its specified range. This finding is likely to enable designers to balance the usage of cement and fly ash from an environmental consideration. By contrast, the strength is sensitive to the binder concentration, and the variation in the binder concentration is found to be amplified in the variation of strength. In this study, the variations in binder concentration and in strength are both linked up with the operational parameter of blade rotation number. The results could benefit engineers in quality control prior to the soil stabilization.

\section{Appendix}

\section{Relationship between Two State Variables and Strength Equation}

Based on the illustrations and definitions in Figure 3, the following relationships can be derived:

$$
\begin{aligned}
& \frac{M_{S}+M_{F}}{M_{C}}=\frac{(1-\alpha)(1+w)}{(1+A) \alpha \beta}+\frac{1-\beta}{\beta}, \\
& \frac{M_{S}+M_{C}}{M_{F}}=\frac{(1-\alpha)(1+w)}{(1+A) \alpha(1-\beta)}+\frac{\beta}{1-\beta},
\end{aligned}
$$




$$
\begin{aligned}
\frac{M_{W 1}+M_{W 2}}{M_{C}} & =\frac{A(1-\alpha)(1+w)}{(1+A) \alpha \beta}+\frac{w}{\beta}, \\
\frac{M_{W 1}+M_{W 2}-0.4 M_{C}}{M_{F}} & =\frac{A(1-\alpha)(1+w)}{(1+A) \alpha(1-\beta)}+\frac{(w-0.4) \beta}{(1-\beta)}+w .
\end{aligned}
$$

By substituting equations (A.1)-(A.4) into equations (2) and (3), the state variables $\alpha$ and $\beta$ can be linked up with the strength prediction equation. For simplicity, the in situ water content $w$ is set constant at $100 \%$, and binder-water ratio of the slurry $A$ is set constant at 1 . By doing so, the average mix ratio is $2: 0.655: 0.345: 3$, being the same as that of the laboratory tests.

\section{Data Availability}

The data used to support the findings of this study are included within the article.

\section{Conflicts of Interest}

The authors declare that there are no conflicts of interest.

\section{Acknowledgments}

This work was supported by the National Natural Science Foundation of China (nos. 71732001 and 52004105) and Fundamental Research Funds for the Central Universities of China (2018KFYYXJJ005). The research fund of Xuzhou Key Research and Development Program (KC20199) is gratefully acknowledged.

\section{References}

[1] Y. Liu, F.-H. Lee, S.-T. Quek, E. J. Chen, and J.-T. Yi, "Effect of spatial variation of strength and modulus on the lateral compression response of cement-admixed clay slab," Géotechnique, vol. 65, no. 10, pp. 851-865, 2015.

[2] H. Xiao, W. H. Shen, and F. H. Lee, "Engineering properties of marine clay admixed with Portland cement and blended cement with siliceous fly ash," Journal of Materials in Civil Engineering, vol. 29, no. 10, 2017.

[3] BSI (British Standards Institution), Cement-Part 1: Composition, Specifications and Conformity Criteria for Common Cements, BS EN 197-1:2000, BSI (British Standards Institution), London, UK, 2011.

[4] H. Xiao, "Evaluating the stiffness of chemically stabilized marine clay," Marine Georesources \& Geotechnology, vol. 35, no. 5, pp. 698-709, 2017.

[5] Q. Cheng, H. Xiao, Y. Liu, W. Wang, and L. Jia, "Primary yielding locus of cement-stabilized marine clay and its applications," Marine Georesources \& Geotechnology, vol. 37, no. 4, pp. $488-505,2019$.

[6] Y. Liu, Y. J. Jiang, H. Xiao, and F. H. Lee, "Determination of representative strength of deep cement-mixed clay from core strength data," Géotechnique, vol. 67, no. 4, pp. 350-364, 2017.

[7] T. Namikawa and J. Koseki, "Effects of spatial correlation on the compression behavior of a cement-treated column," Journal of Geotechnical and Geoenvironmental Engineering, vol. 139, no. 8, pp. 1346-1359, 2013.
[8] Y. Liu, L. Q. He, Y. J. Jiang, M. M. Sun, E. J. Chen, and F.-H. Lee, "Effect of in situ water content variation on the spatial variation of strength of deep cement-mixed clay," Géotechnique, vol. 69, no. 5, pp. 391-405, 2019.

[9] Y. Pan, Y. Liu, J. Hu, M. Sun, and W. Wang, "Probabilistic investigations on the watertightness of jet-grouted ground considering geometric imperfections in diameter and position," Canadian Geotechnical Journal, vol. 54, no. 10, pp. 1447-1459, 2017.

[10] E. J. Chen, Y. Liu, and F.-H. Lee, "A statistical model for the unconfined compressive strength of deep-mixed columns," Géotechnique, vol. 66, no. 5, pp. 351-365, 2016.

[11] Y. Liu, J. Hu, H. Wei, and A.-L. Saw, "A direct simulation algorithm for a class of beta random fields in modelling material properties," Computer Methods in Applied Mechanics and Engineering, vol. 326, pp. 642-655, 2017.

[12] R. Wang, D. Li, M. Wang, and Y. Liu, "Deterministic and probabilistic investigations on piping occurrence during tunnelling through spatially variable soils," ASCE-ASME Journal of Risk and Uncertainty in Engineering Systems, Part A: Civil Engineering, vol. 7, no. 2, 2020.

[13] J. J. Zheng, Y. Liu, Y. T. Pan, and J. Hu, "Statistical evaluation of the load-settlement response of a multi-column composite foundation," International Journal of Geomechanics, vol. 18, no. 4, Article ID 04018015, 2018.

[14] M. Fujii, M. Kawamura, M. Tamura, K. Watanabe, and E. Mizoguchi, "Quality evaluation method for soil-cement column by deep mixing method," Journal of the Society of Materials Science, Japan, vol. 53, no. 1, pp. 9-12, 2004.

[15] S. Larsson, H. Stille, and L. Olsson, "On horizontal variability in lime-cement columns in deep mixing," Géotechnique, vol. 55, no. 1, pp. 33-44, 2005.

[16] E. J. Chen, L. Ding, Y. Liu, X. Ma, and M. J. Skibniewski, “On spectral representation method and Karhunen-Loève expansion in modelling construction material properties," Archives of Civil and Mechanical Engineering, vol. 18, no. 3, pp. 768-783, 2018.

[17] Y. Pan, Y. Liu, and E. J. Chen, "Probabilistic investigation on defective jet-grouted cut-off wall with random geometric imperfections," Géotechnique, vol. 69, no. 5, pp. 420-433, 2019. 